\title{
Pulmonary Absorption of Recombinant Human Granulocyte Colony-Stimulating Factor (rhG-CSF) after Intratracheal Administration to Rats
}

\author{
Minoru Machida, ${ }^{*, a}$ Masahiro HaYashI ${ }^{b}$ and Shoji AwazU ${ }^{c}$ \\ Formulation Technology Lab., Development Div., Chugai Pharmaceutical Co., Ltd., ${ }^{a}$ 5-1, Ukima 5-chome, Kita-ku, \\ Tokyo 115, Japan, Department of Pharmaceutics, Faculty of Pharmaceutical Sciences, Science University of Tokyo, ${ }^{b}$ \\ 12 Ichigaya Funagawara-machi, Shinjuku-ku, Tokyo 162, Japan and Department of Biopharmaceutics, School of \\ Pharmacy, Tokyo University of Pharmacy and Life Science ( formerly Tokyo College of Pharmacy), ${ }^{c}$ 1432-1 Horinouchi, \\ Hachioji, Tokyo 192-03, Japan. Received July 10, 1995; accepted October 19, 1995
}

\begin{abstract}
Pulmonary absorption of recombinant human granulocyte colony-stimulating factor (rhG-CSF) was examined in the rat. The relative bioavailabilities of rhG-CSF after intratracheal administration to intravenous and subcutaneous means were $11.6 \%$ and $27.4 \%$, respectively, which were evaluated from the ratio of area under the curve $(A U C)$ of the plasma rhG-CSF concentration versus time for $8 \mathrm{~h}$. The pharmacological availability after the pulmonary absorption was determined from the $A U C$ for the increase ratio in total blood leukocyte numbers versus time curve. The availability was equal to or more than the availabilities after intravenous or subcutaneous administration. Comparison of the two different parenteral administration routes for rhG-CSF, intratracheal and intranasal showed the pharmacological availability after the intratracheal administration to be superior. The pulmonary absorption of rhG-CSF is thus an effective parenteral route of administration.
\end{abstract}

Key words rhG-CSF; pulmonary absorption; intratracheal administration; total leukocyte number; relative bioavailability; pharmacological availability

Human granulocyte colony-stimulating factor (hGCSF), a hydrophilic glycoprotein of $20000 \mathrm{Da}$ is a hematogenic factor which stimulates granulocyte precursor cells in the bone marrow and specifically promotes their differentiation and proliferation into granulocytes. Recombinant hG-CSF (rhG-CSF) has recently been produced from Chinese hamster ovary cells with high purity and in large quantities. ${ }^{1,2)}$ rhG-CSF is used therapeutically for the treatment of neutropenia that occurs during tumor chemotherapy, for various blood myelodysplasias and for bone marrow transplantation.

Most peptides are so rapidly hydrolyzed and poorly absorbed in the gastrointestinal tract that they are usually administered intravenously or subcutaneously for therapeutic use. As alternative administration routes for physiologically active protein of comparatively low molecular weight such as insulin or calcitonin are nasal, ${ }^{3)}$ buccal ${ }^{4)}{ }^{\text {rectal }}{ }^{5)}$ and ocular ${ }^{6)}$ routes. For proteins of more than $10000 \mathrm{Da}$, however, few trials have been made to develop new routes of administration.

rhG-CSF is currently administered intravenously and subcutaneously, but an other convenient route would be preferable. The authors earlier examined the nasal absorption of rhG-CSF, and reported that the nasal route gave $5-10 \%$ pharmacological availability of the substance, ${ }^{7)}$ that the bioavailability was enhanced three times by Laureth-9 (nonionic surfactant) and that it depended on hydrophilic lipophilic balance (HLB) values of the nonionic surfactants added. ${ }^{8)}$ Niven et al. ${ }^{9)}$ examined the intratracheal route as an alternative and reported that it showed higher availability than the nasal route using nonglycosylated methionyl, Escherichia coliderived rhG-CSF. In this study, the authors report the results after intratracheal administration of glycosylated rhG-CSF, which has higher pharmacological activity than nonglycosylated one, ${ }^{10)}$ to examine the greater efficacy of the pulmonary route than the nasal.

\section{MATERIALS AND METHODS}

Chemicals The chemicals and bulk solution of rhGCSF used in this study were as follows: Tween 20 from Nikko Chemicals Co., Ltd., Tokyo, Japan; sodium pentobarbital (Nembutal ${ }^{\circledR}$ injection, $50 \mathrm{mg} / \mathrm{ml}$ ) from Sankyo Co., Ltd., Tokyo and sodium phenobarbital (Phenobal ${ }^{\circledR}$, $0.1 \mathrm{~g} / \mathrm{ml}$ ) from Dainippon Pharmaceutical Co., Ltd., Osaka, Japan. A bulk solution of rhG-CSF (phosphate buffer solution, $440 \mu \mathrm{g} / \mathrm{ml}$, specific activity $10^{8} \mathrm{U} / \mathrm{mg}$ protein) was supplied by Chugai Pharmaceutical Co., Ltd., Tokyo. Other reagents were of analytical grade or better.

Preparation of rhG-CSF Solution for Intravenous, Subcutaneous and Intratracheal Administration The $\mathrm{pH}$ and osmotic pressure of rhG-CSF solution administered were adjusted to $\mathrm{pH} 4-8$ with $100 \mathrm{~mm} \mathrm{NaH} \mathrm{PO}_{4}$ or $1 \mathrm{~N}$ $\mathrm{HCl}$ and to $180-1140 \mathrm{mOsm} / \mathrm{kg}$ with $\mathrm{NaCl}$. Tween 20 was added to the solution to make a final concentration of $0.01 \%(\mathrm{w} / \mathrm{v})$, and final rhG-CSF concentration was $250 \mu \mathrm{g} / \mathrm{ml}$.

Animal Experiments for Pharmacokinetic Study Male SD rats (8 weeks old, 250-300 g: Clea Japan, Inc., Tokyo) were used.

Femoral artery and vein were cannulated with polyethylene tubing (PE-50, Clay-Adams, U.S.A.). Under the combined anesthesia of sodium phenobarbital $(100 \mathrm{mg} / \mathrm{kg})$ and sodium pentobarbital $(50 \mathrm{mg} / \mathrm{kg})$ administered intraperitoneally, the rhG-CSF solution was administered into lung by the method of Enna and Schanker. ${ }^{11)}$ Intravenous (i.v.) and subcutaneous (s.c.) administration were performed through the femoral vein cannula and into the back skin, respectively, after the animals awakened. Blood samples were collected from the femoral artery for $24 \mathrm{~h}$ after i.v. and s.c. administration, 
but only for $8 \mathrm{~h}$ after intratracheal (i.t.) administration according to Enna and Schanker. ${ }^{11)}$

Animal Experiments for Pharmacodynamic Study The pharmacological activity was determined by the increase of total leukocyte number as reported previously. ${ }^{7}$ All administration and blood sampling for i.v. and s.c. routes were performed as reported previously. ${ }^{7)}$ For i.t. route, drug solution was injected directly into the trachea under ether anesthesia. About $50 \mu \mathrm{l}$ of blood was collected periodically from the tail vein for $72 \mathrm{~h}$ following all three types of administration at 9 a.m. Twenty $\mu$ l of the collected sample was used in the determination of leukocytes.

Assay The plasma concentration of rhG-CSF was determined by enzyme immunoassay. ${ }^{12)}$ Total leukocyte numbers were counted with a microcellcounter (Sysmex F-500, Toa Medical Electronics, Tokyo).

\section{RESULTS}

The plasma concentration time-courses after i.v., s.c. and i.t. administration of $100 \mu \mathrm{g} / \mathrm{kg}$ rhG-CSF are shown in Fig. 1.

The area under the plasma concentration-time curve of rhG-CSF until $8 \mathrm{~h}$ after administration $\left(A U C_{0-8}\right)$, and bioavailability are listed in Table 1 . The relative bioavailability of i.t. administration to i.v. and s.c. were $11.6 \%$ and $27.4 \%$, respectively. The effects of $\mathrm{pH}$ and

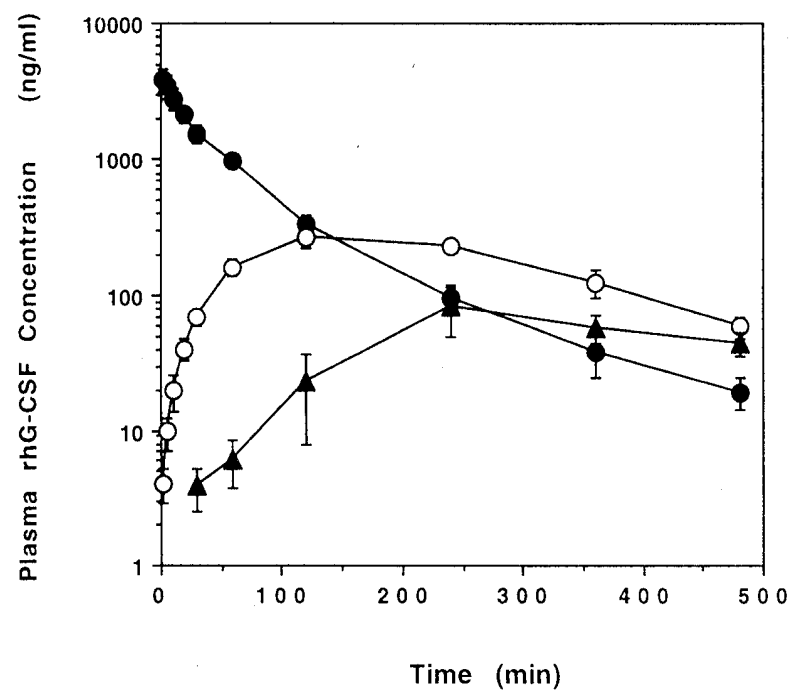

Fig. 1. Plasma Concentration of rhG-CSF Following Intravenous $(\bullet)$, Subcutaneous $(O)$ and Intratracheal $(\boldsymbol{\Delta})$ Administration

Dose of rhG-CSF is $100 \mu \mathrm{g} / \mathrm{kg}$. Values represent the mean \pm S.E. of more than four rats.

Table 1. AUC and Relative Bioavailability after rhG-CSF Administration in Rat

\begin{tabular}{lcc}
\hline $\begin{array}{c}\text { Administration } \\
\text { route }\end{array}$ & $\begin{array}{c}A U C_{0-8} \\
\left(\mathrm{ng} \mathrm{h} \mathrm{ml}^{-1}\right)^{a)}\end{array}$ & $\begin{array}{c}\text { Relative } \\
\text { bioavailability (\%) }\end{array}$ \\
\hline Intravenous (i.v.) & $3150.8 \pm 386.2$ & - \\
Subcutaneous (s.c.) & $1340.1 \pm 209.9$ & - \\
Intratracheal (i.t.) & $366.6 \pm 106.1$ & $11.6 \pm 3.4$ (to i.v.) \\
& & $27.4 \pm 7.9$ (to s.c.)
\end{tabular}

Data are the mean \pm S.E. of more than four rats. a) $A U C$ for $0-8 \mathrm{~h}$ after administration of $100 \mu \mathrm{g} / \mathrm{kg}$ of rhG-CSF. b) Calculated using $A U C_{0-8}$. osmotic pressure of rhG-CSF solution on the bioavailability after i.t. administration are listed in Table 2 .

Under physiological conditions as $\mathrm{pH} 6.5$ or 285 $\mathrm{mOsm} / \mathrm{kg}$, the relative bioavailability tended to be less than that under other conditions, but the significant difference was found only in osmotic effects. The reason for this remains to be further investigated. The time-course of increase ratio of total leukocyte numbers in plasma after i.t. administration with that after i.v. and s.c. administration are shown in Fig. 2 to examine the pharmacological effect of rhG-CSF. The increased number of total leukocytes was determined as a ratio to these numbers before administration of rhG-CSF.

The leukocyte number after saline administration as control varied chronologically but remained constant to a certain extent, showing that the surgical procedure did not affect the leukocyte number. The time-courses of leukocyte number were not as different among the three administration routes as those of rhG-CSF plasma con-

Table 2. Effects of $\mathrm{pH}$ and Osmotic Pressure of rhG-CSF Solution on Relative Bioavailability of Intratracheally Administered rhG-CSF to Intravenous (i.v.) or Subcutaneous (s.c.) Administration in Rat

\begin{tabular}{ccll}
\hline \hline $\mathrm{pH}$ & $\begin{array}{c}\text { Osmotic } \\
\text { pressure } \\
(\mathrm{mOsm} / \mathrm{kg})\end{array}$ & \multicolumn{1}{c}{ Relative bioavailability $(\%)^{a)}$} \\
\hline 4.0 & 285 & $15.3 \pm 5.9$ (to i.v.) & $36.1 \pm 13.8$ (to s.c.) \\
5.0 & 285 & $13.6 \pm 6.3$ & $32.0 \pm 14.8$ \\
6.5 & 180 & $20.2 \pm 5.2^{*}$ & $47.4 \pm 12.1^{*}$ \\
6.5 & 285 & $11.6 \pm 3.4$ & $27.4 \pm 7.9$ \\
6.5 & 560 & $16.7 \pm 7.3$ & $39.3 \pm 17.2$ \\
6.5 & 1140 & $23.3 \pm 8.0^{*}$ & $54.7 \pm 18.9^{*}$ \\
8.0 & 285 & $18.6 \pm 4.4$ & $43.8 \pm 10.5$ \\
\end{tabular}

Data are the mean \pm S.E. of more than four rats. a) Calculated by the ratio of $A U C_{0-8}$ after intratracheal administration to that after intravenous (i.v.) and subctaneous (s.c.) administration of rhG-CSF solution at the corresponding $\mathrm{pH}$ and osmotic pressure. $* 0.01<p<0.05$ vs. the relative bioavailability at $\mathrm{pH} 6.5$ and $285 \mathrm{mOsm} / \mathrm{kg}$.

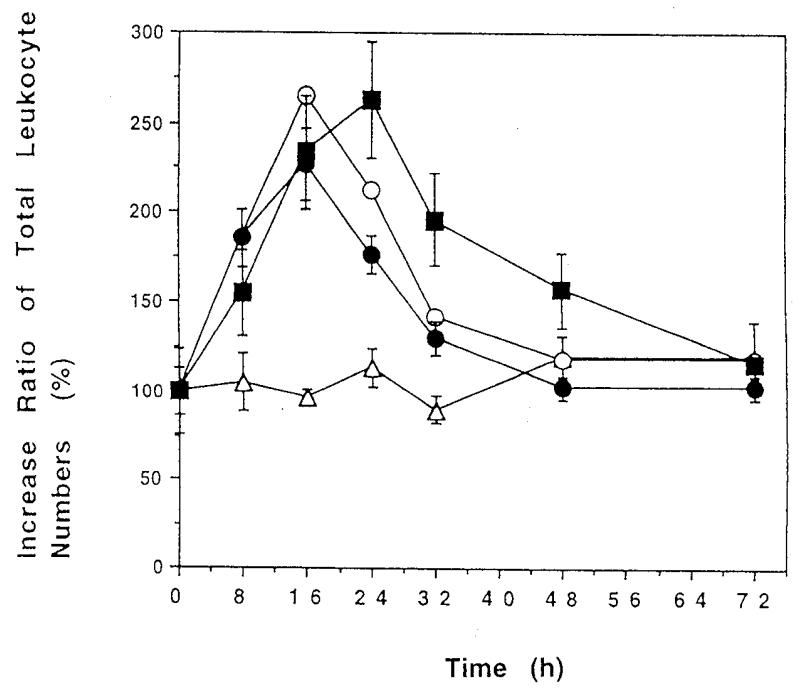

Fig. 2. Increase Ratio of Total Leukocyte Numbers Following Intravenous (O), Subcutaneous (O), and Intratracheal ( $\square$ ) Administration of rhG-CSF

In this control $(\triangle)$, saline solution was administered intratracheally. Values on the ordinate represent the increase ratios of total leukocyte numbers, and $100 \%$ indicates the initial value before administration. Dose of rhG-CSF is $100 \mu \mathrm{g} / \mathrm{kg}$. Data are the mean \pm S.E. of more than four rats. 


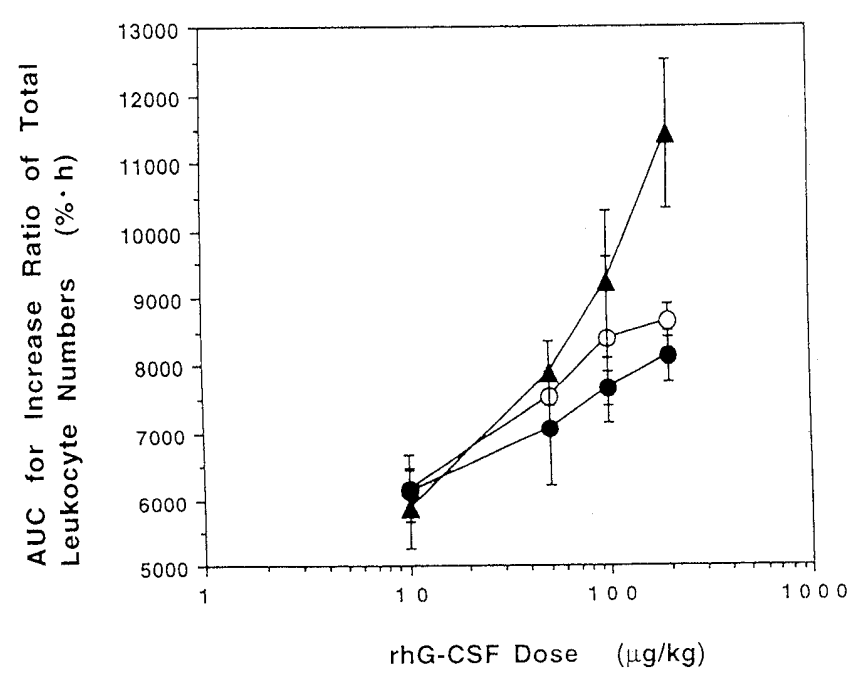

Fig. 3. Relation between $A U C$ for Increase Ratio of Total Leukocyte Numbers and Dose of rhG-CSF Following Intravenous (0), Subcutaneous $(O)$ and Intratracheal $(\boldsymbol{\Delta})$ Administration of rhG-CSF

Data for three different routes are the mean \pm S.E. of more than four rats.

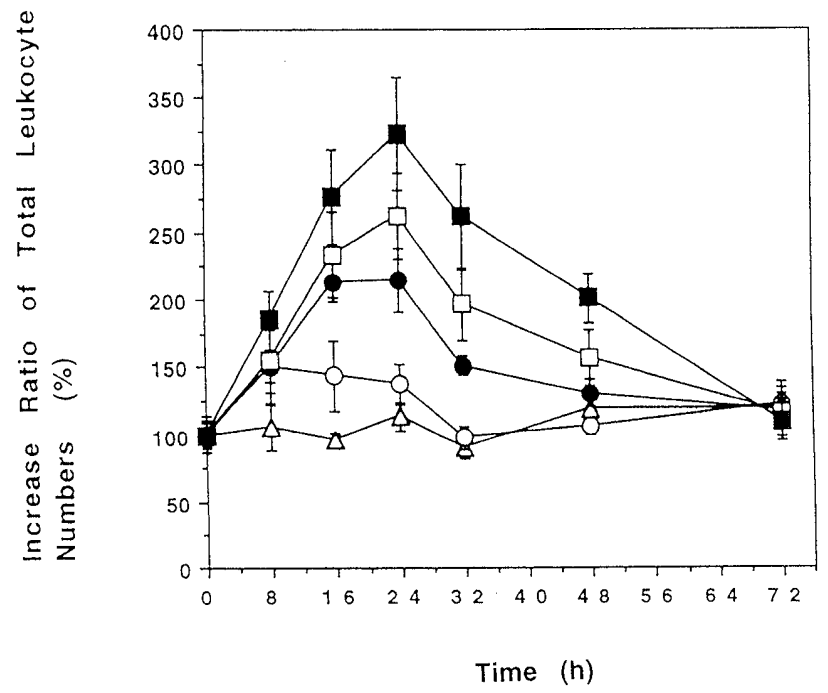

Fig. 4. Change in the Total Leukocyte Numbers Following Intratracheal Administration of rhG-CSF

Doses are $10(\bigcirc), 50(\bigcirc), 100(\square)$ and $200 \mu \mathrm{g} / \mathrm{kg}(\square)$. In the control $(\triangle)$, saline solution was administered intratracheally. Data are the mean \pm S.E. of more than four rats.

centration. The pharmacological effect appeared more slowly and was sustained longer than rhG-CSF plasma concentration by all routes. The pharmacological availabilities after s.c. administration which was calculated as the $A U C$ for the increase ratio vs. time curve to that after i.v. and i.t. administration were $111.1 \pm 15.1 \%$ and $89.9 \pm 12.2 \%$ at $100 \mu \mathrm{g} / \mathrm{kg}$, respectively. The dose dependence of rhG-CSF on leukocyte number differed among the three administration routes as shown in Fig. 3. The i.t. administration showed similar availability to that by s.c. and i.v. at $10-100 \mu \mathrm{g} / \mathrm{kg}$, but at $200 \mu \mathrm{g} / \mathrm{kg}$, the availability after i.t. administration was greatest.

Figure 4 shows the dose effect of rhG-CSF on leukocyte number after i.t. administration. The peak number was observed about $8 \mathrm{~h}$ after the administration of $10 \mu \mathrm{g} / \mathrm{kg}$ rhG-CSF, but at 50-200 $\mu \mathrm{g} / \mathrm{kg}$ administration, the peaks appeared later.

\section{DISCUSSION}

The i.t. administration was shown to be a more effective route for rhG-CSF than intranasal administration, since the relative bioavailability of rhG-CSF after i.t. administration (11.6\% to i.v. administration and $27.4 \%$ to s.c. administration in Table 1) was more than ten times larger than that after intranasal administration $(0.92 \%$ to i.v. administration and $1.95 \%$ to s.c. administration), as reported previously. ${ }^{7)}$ The bioavailability of rhG-CSF after i.t. administration obtained in this report does not contradict those of insulin after i.t. administration $(7-10 \%)$ reported by Wigley et al. ${ }^{13)}$ and $(40 \%)$ by Yoshida et al. ${ }^{14)}$ considering the relation between molecular weight and bioavailability after i.t. administration of FITC-dextran with molecular weight of $4000-70000 .^{15)}$ These improved values for bioavailability show that i.t. administration is an effective route for peptide and protein. Figure 3 shows that although the pharmacological effect of rhG-CSF increased dosedependently, i.t. administration had the highest effect among the three routes. This means that the plasma concentration of rhG-CSF does not correspond to its pharmacological effect, although a dose-dependent pharmacological effect was observed by this route (Fig. 4). Although of the three routes plasma appearance and apparent elimination of rhG-CSF was slowest after i.t. administration (Fig. 1), this tended to result in higher pharmacological activity of rhG-CSF, especially at 200 $\mu \mathrm{g} / \mathrm{kg}$, the activity was significantly greater than that after i.v. or s.c. administration (Fig. 3). These results suggest that the length of time in which rhG-CSF remains in plasma has some relation with the activity, though the mechanism for this is not yet known. From the pharmacological point of view, i.t. administration is suggested to be effective route for current clinical use. Administration by this route is probably worthy of further study, not only to obtain an even greater pharmacological effect but also to develop an invasive administration route assuring a better quality of life of patients.

Acknowledgments The constructive comments of $\mathrm{Mr}$. Kazuo Igusa and Dr. Masayuki Arakawa at Chugai Pharmaceutical Co., Ltd. are gratefully acknowledged. The authors wish to thank Miss Keiko Sano for her excellent technical assistance.

\section{REFERENCES}

1) Nagata S., Tsuchiya M., Asano S., Yamamoto O., Hirata Y., Kubota N., Oheda M., Nomura H., Yamazaki T., EMBO J., 5, 575 (1986).

2) Nagata S., Tsuchiya M., Asano S., Kaziro Y., Yamazaki T., Yamamoto O., Hirai Y., Kubota N., Oheda M., Nomura H., Ono M., Nature (London), 319, 415 (1986).

3) Hirai S., Yashiki T., Mima H., Int. J. Pharmaceut., 9, 165 (1981).

4) IshidaM., Machida Y., Nambu N., Nagai T., Chem. Pharm. Bull., 29, 810 (1981).

5) Nishihata T., Rytting J. H., Kamada A., Higuchi T., Routh M., Caldwell L., J. Pharm. Pharmacol., 35, 148 (1983).

6) Yamamoto A., Luo A. M., Dodda-Kashi S., Lee V. H., J. Pharmacol. Exp. Ther., 249, 249 (1989).

7) Machida M., Sano K., Arakawa M., Hayashi M., Awazu S., Pharm. 
Res., 10, 1372 (1993).

8) Machida M., Sano K., Arakawa M., Hayashi M., Awazu S., Biol. Pharm. Bull., 17, 1375 (1994).

9) Niven R. W., Lott F. D., Cribbs J. M., Pharm. Res., 10, 1604 (1993)

10) Nissen C., Carbonare V. D., Moser Y., Drug Invest., 7, 346 (1994).

11) Enna S. J., Schanker L. S., Am. J. Physiol., 222, 409 (1972).

12) Motojima H., Kobayashi T., Shimane M., Kamachi S., Fukushima
M., J. Immunol. Methods, 118, 187 (1989).

13) Wigley F. M., Londono J. H., Wood S. H., Shipp J. C., Waldman R. H., Diabetes, 20, 552 (1971).

14) Yoshida H., Okumura K., Hori K., Anmo T., Yamaguchi H., $J$. Pharm. Sci., 68, 670 (1979).

15) Morita T., Yamamoto A., Hashida M., Sezaki H., Biol. Pharm. Bull., 16, 259 (1993). 